\title{
The Spectral Structure of the Electronic Black Box Hamiltonian
}

\author{
P. Grech ${ }^{1,2}$, V. Jakšić ${ }^{1}$, M. Westrich ${ }^{1}$ \\ ${ }^{1}$ Department of Mathematics and Statistics \\ McGill University \\ 805 Sherbrooke Street West \\ Montreal, QC, H3A 2K6, Canada \\ ${ }^{2}$ Centre de recherches mathématiques \\ Université de Montréal \\ 2920 Chemin de la tour \\ Montréal, QC, H3T 1J4, Canada
}

August 11, 2012

\begin{abstract}
We give results on the absence of singular continuous spectrum of the one-particle Hamiltonian underlying the electronic black box model.
\end{abstract}

\section{Introduction and Main Results}

We consider a quantum system $S$ with finite dimensional Hilbert space $\mathcal{H}_{S}$ coupled to a left and a right reservoir with Hilbert spaces $\mathcal{H}_{\ell}, \mathcal{H}_{r}$ respectively. The Hilbert space of the compound system is given by

$$
\mathcal{H}:=\mathcal{H}_{\ell} \oplus \mathcal{H}_{S} \oplus \mathcal{H}_{r}
$$

It carries a free dynamics given by the non-interacting Hamiltonian

$$
H_{0}:=H_{\ell}+H_{S}+H_{r},
$$

where $H_{j}$ is a self-adjoint operator on $\mathcal{H}_{j}$, for $j=\ell, S, r$. The coupling between system and reservoirs is modelled as follows. Let $\chi_{\ell / r} \in \mathcal{H}_{\ell / r}, \delta_{\ell / r} \in \mathcal{H}_{S}$, be distinguished non-zero vectors. The full Hamiltonian is then defined as

$$
H_{\lambda, \nu}:=H_{0}+\lambda\left[\left(\chi_{\ell}, \cdot\right) \delta_{\ell}+\left(\delta_{\ell}, \cdot\right) \chi_{\ell}\right]+\nu\left[\left(\chi_{r}, \cdot\right) \delta_{r}+\left(\delta_{r}, \cdot\right) \chi_{r}\right],
$$

where $\lambda, \nu \in \mathbb{R}$ are control parameters. In the present article we address the question of when the singular continuous spectrum $\sigma_{\mathrm{sc}}\left(H_{\lambda, \nu}\right)$ is empty. 
The fermionic second quantization of the Hamiltonian (1.1) leads to the simplest nontrivial example of the electronic black box model, which has been one of the basic paradigms in the recent developments in non-equilibrium statistical mechanics (see [AJJP1, AJPP2, JKP, N] for references and additional information). The motivation for this article comes from the fact that the absence of singular continuous spectrum is crucial for the rigorous derivation of the Landauer-Büttiker formula in transport theory $[\mathrm{AJPP} 2, \mathrm{~N}]$.

It is not hard to see that the cyclic space spanned by $\left(\chi_{\ell}, \chi_{r}, \delta_{\ell}, \delta_{r}\right)$ and $H_{0}$ agrees with the one spanned by the same vectors and $H_{\lambda, \nu}$. Since $H_{\lambda, \nu}=H_{0}$ on the orthogonal complement of this cyclic space, for our purposes we may assume without loss of generality that $\left(\chi_{\ell}, \chi_{r}, \delta_{\ell}, \delta_{r}\right)$ is a cyclic family for $H_{0}$.

Before formulating our main results we gather the following general facts about the connection between boundary values of analytic functions and the spectral measure $\mu_{\varphi}^{(\lambda, \nu)}$ of $H_{\lambda, \nu}$ and $\varphi$ (see, e.g., [J]). In what follows the quantifier a.e. stands for almost every with respect to Lebesgue measure $\mathcal{L}$ on $\mathbb{R}$.

The Borel transform of $\mu_{\varphi}^{(\lambda, \nu)}$,

$$
G_{\lambda, \nu}(\varphi, \varphi, z):=\int_{\mathbb{R}} \frac{d \mu_{\varphi}^{(\lambda, \nu)}(E)}{E-z},
$$

where $z \in \mathbb{C} \backslash \mathbb{R}$ and $\varphi=\chi_{\ell / r}, \delta_{\ell / r}$, has the following properties:

1. The limit

$$
G_{\lambda, \nu}(\varphi, \varphi, E+\mathrm{i} 0):=\lim _{\epsilon \backslash 0} G_{\lambda, \nu}(\varphi, \varphi, E+i \epsilon),
$$

exists for a.e. $E \in \mathbb{R}$. Moreover, $G_{\lambda, \nu}(\varphi, \varphi, E+\mathrm{i} 0)$ is finite and non-zero for a.e. $E \in \mathbb{R}$.

2. The absolutely continuous part of $\mu_{\varphi}^{(\lambda, \nu)}$ satisfies

$$
d \mu_{\varphi, \text { ac }}^{(\lambda, \nu)}(E)=\frac{1}{\pi} \operatorname{Im} G_{\lambda, \nu}(\varphi, \varphi, E+i 0) d E .
$$

3. The singular part of $\mu_{\varphi}^{(\lambda, \nu)}$ is concentrated on the set

$$
\left\{E \in \mathbb{R} \mid \lim _{\epsilon \backslash 0} \operatorname{Im} G_{\lambda, \nu}(\varphi, \varphi, E+i \epsilon)=\infty\right\}
$$

The connection with spectral theory is established by the spectral theorem through the formula

$$
G_{\lambda, \nu}(\varphi, \varphi, z)=\left(\varphi,\left(H_{\lambda, \nu}-z\right)^{-1} \varphi\right) .
$$

More generally, we write

$$
G_{\lambda, \nu}(\varphi, \psi, z)=\left(\varphi,\left(H_{\lambda, \nu}-z\right)^{-1} \psi\right)
$$

with $\varphi, \psi=\chi_{\ell / r}, \delta_{\ell / r}$. 
Throughout this article we shall assume that

$$
G_{0}\left(\delta_{\ell}, \delta_{r}, E\right)=\left(\delta_{\ell},\left(H_{S}-E\right)^{-1} \delta_{r}\right) \not \equiv 0
$$

(double zero indices will be written as a single index, e.g. $G_{0,0}=G_{0}, \mu_{\varphi}^{(0,0)}=\mu_{\varphi}^{(0)}$, etc.). An equivalent formulation of (1.2) is that the cyclic spaces spanned by $\delta_{\ell / r}$ and $H_{S}$ are not orthogonal and hence that the system $S$ does not trivially decouple into two non-interacting subsystems. The case where $G_{0}\left(\delta_{\ell}, \delta_{r}, E\right) \equiv 0$ is simpler and can be treated by the same techniques. However this case has no relevance for applications to non-equilibrium statistical mechanics that motivated the present work.

To be able to formulate our hypotheses we define

$$
\mathcal{M}_{0}:=\left\{E \in \mathbb{R} \mid G_{0}\left(\chi_{\ell / r}, \chi_{\ell / r}, E+i 0\right) \text { is finite and non-zero }\right\} .
$$

In view of Property $1, \mathcal{L}\left(\mathcal{M}_{0}^{c}\right)=0\left(A^{c}\right.$ denotes the complement of a set $\left.A\right)$. We also define

$$
\mathcal{M}_{\ell / r}:=\left\{E \in \mathcal{M}_{0} \mid 0<\operatorname{Im} G_{0}\left(\chi_{\ell / r}, \chi_{\ell / r}, E+i 0\right)<\infty\right\} .
$$

The set $\mathcal{M}_{\ell} \cup \mathcal{M}_{r}$ is an essential support of the absolutely continuous spectrum of $H_{\lambda, \nu}$ for all $\lambda, \nu \in$ $\mathbb{R}$ (to avoid confusion we recall that the essential support of a.c. spectrum is usually defined as an equivalence class of Borel sets with respect to the relation $\left.B_{1} \sim B_{2} \Leftrightarrow \mathcal{L}\left(\left(B_{1} \backslash B_{2}\right) \cup\left(B_{2} \backslash B_{1}\right)\right)=0\right)$.

To fix notation we also recall that the projection-valued measure corresponding to $H_{\lambda, \nu}$ has a unique decomposition into absolutely continuous, pure point, and singular continuous part,

$$
\mathbf{1}_{B}\left[H_{\lambda, \nu}\right]=\mathbf{1}_{B}^{\mathrm{ac}}\left[H_{\lambda, \nu}\right]+\mathbf{1}_{B}^{\mathrm{pp}}\left[H_{\lambda, \nu}\right]+\mathbf{1}_{B}^{\mathrm{sc}}\left[H_{\lambda, \nu}\right],
$$

where $B \subset \mathbb{R}$ is a Borel set. Our first result reads as follows:

Theorem 1.1 Let $B \subset \mathbb{R}$ be a Borel set such that

$$
\left(\mathcal{M}_{\ell} \cup \mathcal{M}_{r}\right)^{c} \cap B
$$

is countable. Then $\mathbf{1}_{B}^{\mathrm{sc}}\left[H_{\lambda, \nu}\right]=0$ for all $\lambda, \nu$.

Theorem 1.1 has a relatively short proof and can be generalized in various ways via an application of the Feshbach method (see [BFS, DJ]). In the stated form however it contrasts instructively our main result, which has a considerably more technical proof.

Theorem 1.2 Let $B \subset \mathbb{R}$ be a Borel set such that

$$
\mathcal{L}\left(\left(\mathcal{M}_{\ell} \cup \mathcal{M}_{r}\right)^{c} \cap B\right)=0 .
$$

Then $\mathbf{1}_{B}^{\mathrm{sc}}\left[H_{\lambda, \nu}\right]=0$ for a.e. $\lambda, \nu$.

Remark. This result can be viewed as an extension of the Simon-Wolff theorems [SW, J, JKP] to the electronic black box Hamiltonian setting.

Since $H_{\lambda, \nu}-H_{0}$ is compact it follows from Weyl's theorem that $\sigma\left(H_{\lambda, \nu}\right) \backslash \sigma\left(H_{0}\right)$ is countable and hence $\mathbf{1}_{\sigma\left(H_{0}\right)^{c}}^{\mathrm{sc}}\left[H_{\lambda, \nu}\right]=0$. Thus we may formulate as a direct consequence of Theorems 1.1 and 1.2: 
Corollary 1.3 (1) If $\left(\mathcal{M}_{\ell} \cup \mathcal{M}_{r}\right)^{c} \cap \sigma\left(H_{0}\right)$ is countable, then $\sigma_{\mathrm{sc}}\left(H_{\lambda, \nu}\right)=\emptyset$ for all $\lambda, \nu$.

(2) If $\mathcal{L}\left(\left(\mathcal{M}_{\ell} \cup \mathcal{M}_{r}\right)^{c} \cap \sigma\left(H_{0}\right)\right)=0$, then $\sigma_{\mathrm{sc}}\left(H_{\lambda, \nu}\right)=\emptyset$ for a.e. $\lambda, \nu$.

The key ingredient for the proof of Theorem 1.2 and our principal technical result concerns spectral averaging for rank two perturbations. Let $\bar{\mu}_{\varphi}^{(\kappa)}$ be a Borel measure on $\mathbb{R}$ defined by

$$
\bar{\mu}_{\varphi}^{(\kappa)}(B):=\int_{\mathbb{R}} \mu_{\varphi}^{\left(\kappa, \kappa^{\prime}\right)}(B) d \kappa^{\prime}
$$

where $\kappa=\nu$ and $\kappa^{\prime}=\lambda$ if $\varphi=\chi_{\ell}, \delta_{\ell}$, and $\kappa=\lambda$ and $\kappa^{\prime}=\nu$ if $\varphi=\chi_{r}, \delta_{r}$.

Theorem 1.4 (Spectral averaging) There is a finite $\operatorname{set} \mathcal{N} \subset \mathbb{R}$, independent of $\lambda, \nu$, such that for a.e. $\left.\kappa, \bar{\mu}_{\varphi}^{(\kappa)}\right\rceil_{\mathbb{R} \backslash \mathcal{N}}$ is absolutely continuous with respect to $\mathcal{L} \Upsilon_{\mathbb{R} \backslash \mathcal{N}}$.

Remark 1. Spectral averaging for rank one perturbations is a classical result that has been known for a long time and refers to the following surprising fact. Given any self-operator $A_{0}$ on a Hilbert space $\mathcal{H}$ and a unit vector $\psi \in \mathcal{H}$, the spectral measure $\mu_{\psi}^{(\lambda)}$ for $A_{\lambda}:=A_{0}+\lambda(\psi, \cdot) \psi$ averages to the Lebesgue measure:

$$
\bar{\mu}_{\psi}(B)=\int_{\mathbb{R}} \mu_{\psi}^{(\lambda)}(B) d \lambda=\mathcal{L}(B) .
$$

The proof of rank one spectral averaging is simple and can be found in many places in the literature (see, e.g., $[\mathrm{J}, \mathrm{S}])$.

Remark 2. The set $\mathcal{N}$ need not be empty. Let

$$
\mathcal{H}_{\ell}=\mathcal{H}_{r}=L^{2}([-2,-1] \cup[1,2], d x)
$$

and $H_{\ell}=H_{r}$ be the operator of multiplication by the variable $x, \chi_{\ell}=\chi_{r}=\mathbb{1}(\mathbb{1}(x)=1)$, and let $\mathcal{H}_{S}=\mathbb{C}, H_{S}=0, \delta_{\ell}=\delta_{r}=\delta=1$. Note that $\left(\delta, \chi_{\ell}, \chi_{r}\right)$ is a cyclic family for $H_{0}$ and that Theorem 1.4 holds for $H_{\lambda, \nu}$. Let

$$
\psi_{\lambda, \nu}:=\left(-\frac{\lambda}{x}\right) \oplus 1 \oplus\left(-\frac{\nu}{x}\right)
$$

Then $\psi_{\lambda, \nu} \in \mathcal{H}$ and $H_{\lambda, \nu} \psi_{\lambda, \nu}=0$ for all $\lambda, \nu$. The vector $\psi_{\lambda, \nu}$ is orthogonal to the cyclic subspace spanned by $H_{\lambda, \nu}$ and $\left(\chi_{\ell}, \chi_{r}\right)$ and so $\mu_{\delta}^{(\lambda, \nu)}(\{0\})>0$ for all $\lambda, \nu$. This implies

$$
\bar{\mu}_{\delta}^{(\lambda)}(\{0\})=\bar{\mu}_{\delta}^{(\nu)}(\{0\})>0,
$$

for all $\lambda, \nu$ and the averaged measures $\bar{\mu}_{\delta}^{(\kappa)}$ are not absolutely continuous with respect to $\mathcal{L}$.

Remark 3. With an additional argument one can show that $\mathcal{N} \subset \sigma\left(H_{S}\right)$.

As we have already remarked, Theorem 1.1 can be generalized in many ways by application of standard techniques centered around the Feshbach formula. This is not the case with Theorem 1.2. Our proof is essentially restricted to the simplest example (1.1) of the electronic black box Hamiltonian and many interesting questions remain open.

Acknowledgment. The research of V.J. was partly supported by NSERC. 


\section{Proofs}

\subsection{Basic formulas}

Lemma 2.1 For $z \in \mathbb{C} \backslash \mathbb{R}$,

$G_{\lambda, \nu}\left(\delta_{\ell}, \delta_{\ell}, z\right)=\frac{1}{D(z)}\left[\left(1-\nu^{2} G_{0}\left(\chi_{r}, \chi_{r}, z\right) G_{0}\left(\delta_{r}, \delta_{r}, z\right)\right) G_{0}\left(\delta_{\ell}, \delta_{\ell}, z\right)+\nu^{2} G_{0}\left(\chi_{r}, \chi_{r}, z\right) G_{0}\left(\delta_{\ell}, \delta_{r}, z\right) G_{0}\left(\delta_{r}, \delta_{\ell}, z\right)\right]$

and

$$
G_{\lambda, \nu}\left(\chi_{\ell}, \chi_{\ell}, z\right)=\frac{1}{D(z)}\left[G_{0}\left(\chi_{\ell}, \chi_{\ell}, z\right)\left(1-\nu^{2} G_{0}\left(\chi_{r}, \chi_{r}, z\right) G_{0}\left(\delta_{r}, \delta_{r}, z\right)\right)\right]
$$

where

$$
\begin{aligned}
D(z)=( & \left.-\nu^{2} G_{0}\left(\chi_{r}, \chi_{r}, z\right) G_{0}\left(\delta_{r}, \delta_{r}, z\right)\right)\left(1-\lambda^{2} G_{0}\left(\chi_{\ell}, \chi_{\ell}, z\right) G_{0}\left(\delta_{\ell}, \delta_{\ell}, z\right)\right) \\
& -\nu^{2} \lambda^{2} G_{0}\left(\chi_{r}, \chi_{r}, z\right) G_{0}\left(\chi_{\ell}, \chi_{\ell}, z\right) G_{0}\left(\delta_{\ell}, \delta_{r}, z\right) G_{0}\left(\delta_{r}, \delta_{\ell}, z\right) .
\end{aligned}
$$

Proof. The second resolvent identity

$$
\left(H_{\lambda, \nu}-z\right)^{-1}=\left(H_{0}-z\right)^{-1}-\left(H_{\lambda, \nu}-z\right)^{-1}\left(H_{\lambda, \nu}-H_{0}\right)\left(H_{0}-z\right)^{-1}
$$

leads to the system of equations

$$
\begin{aligned}
G_{\lambda, \nu}\left(\delta_{\ell}, \delta_{\ell}, z\right) & =G_{0}\left(\delta_{\ell}, \delta_{\ell}, z\right)-\left[\lambda G_{\lambda, \nu}\left(\delta_{\ell}, \chi_{\ell}, z\right) G_{0}\left(\delta_{\ell}, \delta_{\ell}, z\right)+\nu G_{\lambda, \nu}\left(\delta_{\ell}, \chi_{r}, z\right) G_{0}\left(\delta_{r}, \delta_{\ell}, z\right)\right], \\
G_{\lambda, \nu}\left(\delta_{\ell}, \chi_{\ell}, z\right) & =-\lambda G_{\lambda, \nu}\left(\delta_{\ell}, \delta_{\ell}, z\right) G_{0}\left(\chi_{\ell}, \chi_{\ell}, z\right), \\
G_{\lambda, \nu}\left(\delta_{\ell}, \chi_{r}, z\right) & =-\nu G_{\lambda, \nu}\left(\delta_{\ell}, \delta_{r}, z\right) G_{0}\left(\chi_{r}, \chi_{r}, z\right), \\
G_{\lambda, \nu}\left(\delta_{\ell}, \delta_{r}, z\right) & =G_{0}\left(\delta_{\ell}, \delta_{r}, z\right)-\left[\lambda G_{\lambda, \nu}\left(\delta_{\ell}, \chi_{\ell}, z\right) G_{0}\left(\delta_{\ell}, \delta_{r}, z\right)+\nu G_{\lambda, \nu}\left(\delta_{\ell}, \chi_{r}, z\right) G_{0}\left(\delta_{r}, \delta_{r}, z\right)\right] .
\end{aligned}
$$

Solving the system one derives the formula for $G_{\lambda, \nu}\left(\delta_{\ell}, \delta_{\ell}, z\right)$. Similarly,

$$
\begin{aligned}
& G_{\lambda, \nu}\left(\chi_{\ell}, \chi_{\ell}, z\right)=G_{0}\left(\chi_{\ell}, \chi_{\ell}, z\right)-\lambda G_{\lambda, \nu}\left(\chi_{\ell}, \delta_{\ell}, z\right) G_{0}\left(\chi_{\ell}, \chi_{\ell}, z\right), \\
& G_{\lambda, \nu}\left(\chi_{\ell}, \delta_{\ell}, z\right)=-\nu G_{\lambda, \nu}\left(\chi_{\ell}, \chi_{r}, z\right) G_{0}\left(\delta_{r}, \delta_{\ell}, z\right)-\lambda G_{\lambda, \nu}\left(\chi_{\ell}, \chi_{\ell}, z\right) G_{0}\left(\delta_{\ell}, \delta_{\ell}, z\right), \\
& G_{\lambda, \nu}\left(\chi_{\ell}, \chi_{r}, z\right)=-\nu G_{\lambda, \nu}\left(\chi_{\ell}, \delta_{r}, z\right) G_{0}\left(\chi_{r}, \chi_{r}, z\right), \\
& G_{\lambda, \nu}\left(\chi_{\ell}, \delta_{r}, z\right)=-\nu G_{\lambda, \nu}\left(\chi_{\ell}, \chi_{r}, z\right) G_{0}\left(\delta_{r}, \delta_{r}, z\right)-\lambda G_{\lambda, \nu}\left(\chi_{\ell}, \chi_{\ell}, z\right) G_{0}\left(\delta_{\ell}, \delta_{r}, z\right),
\end{aligned}
$$

and the formula for $G_{\lambda, \nu}\left(\chi_{\ell}, \chi_{\ell}, z\right)$ follows. 


\subsection{Proof of Theorem 1.1}

Let

$$
\mathcal{S}:=\left\{E \in \mathbb{R} \mid G_{0}\left(\delta_{\ell}, \delta_{r}, E\right)=0\right\} .
$$

The Condition (1.2) ensures that $\mathcal{S}$ is a finite set. Recall also our standing assumption that $\left(\chi_{\ell}, \chi_{r}, \delta_{\ell}, \delta_{r}\right)$ is a cyclic family for $H_{0}$ and hence for $H_{\lambda, \nu}$ for all $\lambda, \nu$. Thus, to prove Theorem 1.1 it suffices to show that for $E \in\left(\mathcal{M}_{\ell} \cup \mathcal{M}_{r}\right) \backslash\left(\sigma\left(H_{S}\right) \cup \mathcal{S}\right)$ and $\varphi=\chi_{\ell / r}, \delta_{\ell / r}$, the limits

$$
\lim _{\epsilon \searrow 0} \operatorname{Im} G_{\lambda, \nu}(\varphi, \varphi, E+\mathrm{i} \epsilon)
$$

exist and are finite for all $\lambda, \nu$.

If $\lambda=\nu=0$ there is nothing to prove and hence we may assume that at least one of the parameters is non-zero. By symmetry it suffices to consider the cases $\varphi=\chi_{\ell}, \delta_{\ell}$. Finally, it follows from the definition of $\mathcal{M}_{\ell / r}$ and Lemma 2.1 that it suffices to show that for $E \in\left(\mathcal{M}_{\ell} \cup \mathcal{M}_{r}\right) \backslash\left(\sigma\left(H_{S}\right) \cup \mathcal{S}\right)$ we have $D(E) \neq 0$, where

$$
\begin{aligned}
D(E) & =\left(1-\nu^{2} G_{0}\left(\chi_{r}, \chi_{r}, E+i 0\right) G_{0}\left(\delta_{r}, \delta_{r}, E\right)\right)\left(1-\lambda^{2} G_{0}\left(\chi_{\ell}, \chi_{\ell}, E\right) G_{0}\left(\delta_{\ell}, \delta_{\ell}, E\right)\right) \\
& -\nu^{2} \lambda^{2} G_{0}\left(\chi_{r}, \chi_{r}, E+i 0\right) G_{0}\left(\chi_{\ell}, \chi_{\ell}, E+i 0\right) G_{0}\left(\delta_{\ell}, \delta_{r}, E\right) G_{0}\left(\delta_{r}, \delta_{\ell}, E\right) .
\end{aligned}
$$

We argue by contradiction. Suppose that $D(E)=0$ for some $E \in\left(\mathcal{M}_{\ell} \cup \mathcal{M}_{r}\right) \backslash\left(\sigma\left(H_{S}\right) \cup \mathcal{S}\right)$. Set $a=G_{0}\left(\delta_{\ell}, \delta_{\ell}, E\right), b=G_{0}\left(\delta_{r}, \delta_{r}, E\right), d=G_{0}\left(\delta_{\ell}, \delta_{\ell}, E\right) G_{0}\left(\delta_{r}, \delta_{r}, E\right)-G_{0}\left(\delta_{\ell}, \delta_{r}, E\right) G_{0}\left(\delta_{\ell}, \delta_{r}, E\right)$. Since

$$
G_{0}\left(\delta_{\ell}, \delta_{r}, E\right) G_{0}\left(\delta_{r}, \delta_{\ell}, E\right)=\left|G_{0}\left(\delta_{\ell}, \delta_{r}, E\right)\right|^{2},
$$

the numbers $a, b, d$ are real. We also set $l=G_{0}\left(\chi_{\ell}, \chi_{\ell}, E+i 0\right), r=G_{0}\left(\chi_{r}, \chi_{r}, E+i 0\right)$. Then the relation $D(E)=0$ can be written as

$$
1-\lambda^{2} a l-\nu^{2} b r+\lambda^{2} \nu^{2} d r l=0
$$

or equivalently, as

$$
1-\nu^{2} b r=\lambda^{2} l\left(a-\nu^{2} d r\right) .
$$

Multiplying both sides of (2.4) with $a-\nu^{2} d \bar{r}$ yields

$$
a-\nu^{2} d \bar{r}-\nu^{2} b a r+\nu^{4} b d|r|^{2}=\lambda^{2} l\left|a-\nu^{2} d r\right|^{2} .
$$

Taking imaginary parts we derive

$$
\nu^{2} d \operatorname{Im} r-\nu^{2} b a \operatorname{Im} r=\lambda^{2} \operatorname{Im} l\left|a-\nu^{2} d r\right|^{2} .
$$

The last equation is equivalent to

$$
-\nu^{2}\left|G_{0}\left(\delta_{\ell}, \delta_{r}, E\right)\right|^{2} \operatorname{Im} G_{0}\left(\chi_{r}, \chi_{r}, E+i 0\right)=\lambda^{2} \operatorname{Im} G_{0}\left(\chi_{\ell}, \chi_{\ell}, E+i 0\right)\left|a-\nu^{2} d G_{0}\left(\chi_{r}, \chi_{r}, E+i 0\right)\right|^{2} \text {. }
$$

By symmetry we obtain in addition

$$
-\lambda^{2}\left|G_{0}\left(\delta_{\ell}, \delta_{r}, E\right)\right|^{2} \operatorname{Im} G_{0}\left(\chi_{\ell}, \chi_{\ell}, E+i 0\right)=\nu^{2} \operatorname{Im} G_{0}\left(\chi_{r}, \chi_{r}, E+i 0\right)\left|b-\lambda^{2} d G_{0}\left(\chi_{\ell}, \chi_{\ell}, E+i 0\right)\right|^{2} \text {. }
$$

The right hand sides of Equations $(2.5,2.6)$ are non-negative, whereas at least one of the left hand sides is strictly negative which is a contradiction. 


\subsection{Proof of Theorem 1.2}

It suffices to show that, for a.e. $\lambda, \nu, \mathbf{1}_{A}\left[H_{\lambda, \nu}\right] \varphi=0$ for the Lebesgue zero set $A:=\left(\mathcal{M}_{\ell} \cup \mathcal{M}_{r}\right)^{c} \cap B \backslash \mathcal{N}$ and $\varphi=\delta_{\ell / r}, \chi_{\ell / r}$. This however follows from Theorem 1.4 since for a.e. $\kappa\left(\kappa=\nu\right.$ and $\kappa^{\prime}=\lambda$ if $\varphi=\chi_{\ell}, \delta_{\ell} ; \kappa=\lambda$ and $\kappa^{\prime}=\nu$ if $\varphi=\chi_{r}, \delta_{r}$ ) we have

$$
0=\bar{\mu}_{\varphi}^{(\kappa)}(A)=\int_{\mathbb{R}} \mu_{\varphi}^{\left(\kappa, \kappa^{\prime}\right)}(A) d \kappa^{\prime}=\int_{\mathbb{R}}\left\|\mathbf{1}_{A}\left[H_{\kappa, \kappa^{\prime}}\right] \varphi\right\|^{2} d \kappa^{\prime} .
$$

\subsection{Proof of Theorem 1.4}

Throughout the proof we shall omit standard measurability arguments (they can be found, for example, in the lecture notes [J]).

We start with some preliminaries. By the symmetry $\ell \leftrightarrow r$ it is sufficient to consider the case $\kappa=\nu$, $\varphi=\chi_{\ell}, \delta_{\ell}$. The singular part of $\mu_{\varphi}^{(\lambda, \nu)}$, denoted $\mu_{\varphi, \text { sing }}^{(\lambda, \nu)}$, is concentrated on the set

$$
S_{\varphi}\left(H_{\lambda, \nu}\right):=\left\{E \in \mathbb{R} \mid \lim _{\epsilon \backslash 0} \operatorname{Im} G_{\lambda, \nu}(\varphi, \varphi, E+i \epsilon)=\infty\right\}
$$

The Poisson transform of the averaged spectral measure $\bar{\mu}_{\varphi}^{(\nu)}$ can be computed by the residue theorem,

$$
\begin{aligned}
P_{\varphi}^{(\nu)}(E+i \epsilon) & :=\int_{\mathbb{R}} \operatorname{Im} G_{\lambda, \nu}(\varphi, \varphi, E+i \epsilon) d \lambda \\
& =\int_{\mathbb{R}} \operatorname{Im}\left[\frac{G_{0, \nu}(\varphi, \varphi, E+i \epsilon)}{1-\lambda^{2} G_{0, \nu}(\varphi, \varphi, E+i \epsilon) G_{0, \nu}(\psi, \psi, E+i \epsilon)}\right] d \lambda \\
& =\operatorname{Im}\left(i \pi \sqrt{\frac{G_{0, \nu}(\varphi, \varphi, E+i \epsilon)}{G_{0, \nu}(\psi, \psi, E+i \epsilon)}}\right) \geq 0,
\end{aligned}
$$

where the last inequality in (2.7) fixes the branch of the square root and $\varphi, \psi \in\left\{\chi_{\ell}, \delta_{\ell}\right\}, \varphi \neq \psi$. The singular part $\bar{\mu}_{\varphi, \text { sing }}^{(\nu)}$ of the averaged spectral measure is concentrated on the set

$$
\bar{S}_{\varphi}^{(\nu)}:=\left\{E \in \mathbb{R} \mid \lim _{\epsilon \backslash 0} P_{\varphi}^{(\nu)}(E+i \epsilon)=\infty\right\}
$$

Since

we have

$$
\bar{\mu}_{\varphi, \operatorname{sing}}^{(\nu)} \leq \int_{\mathbb{R}} \mu_{\varphi, \operatorname{sing}}^{(\lambda, \nu)} d \lambda
$$

$$
\bar{S}_{\varphi}^{(\nu)} \subset \bigcup_{\lambda \in \mathbb{R} \backslash\{0\}} S_{\varphi}\left(H_{\lambda, \nu}\right)
$$

We also introduce the sets

$$
C_{\varphi, \psi}^{(\nu)}:=\left\{E \in \mathbb{R}\left|\lim _{\epsilon \searrow 0}\right| G_{0, \nu}(\varphi, \varphi, E+i \epsilon) \mid=\infty, \lim _{\epsilon \searrow 0} G_{0, \nu}(\psi, \psi, E+i \epsilon)=0\right\}
$$

for $\varphi, \psi \in\left\{\chi_{\ell}, \delta_{\ell}\right\}, \varphi \neq \psi$. Clearly, $\mathcal{L}\left(C_{\varphi, \psi}^{(\nu)}\right)=0$. 


\section{Lemma 2.2}

$$
\overline{\mathcal{S}}_{\varphi}^{(\nu)} \subset C_{\varphi, \psi}^{(\nu)}
$$

Remark. Since $G_{0, \nu}\left(\chi_{\ell}, \chi_{\ell}, E+i \epsilon\right)=G_{0}\left(\chi_{\ell}, \chi_{\ell}, E+i \epsilon\right)$, we have in particular

$$
\begin{aligned}
& \overline{\mathcal{S}}_{\chi_{\ell}}^{(\nu)} \subset\left\{E \in \mathbb{R}\left|\lim _{\epsilon \backslash 0}\right| G_{0}\left(\chi_{\ell}, \chi_{\ell}, E+i \epsilon\right) \mid=\infty\right\}, \\
& \overline{\mathcal{S}}_{\delta_{\ell}}^{(\nu)} \subset\left\{E \in \mathbb{R} \mid \lim _{\epsilon \backslash 0} G_{0}\left(\chi_{\ell}, \chi_{\ell}, \varphi, E+i \epsilon\right)=0\right\} .
\end{aligned}
$$

Proof. Let $E \in \overline{\mathcal{S}}_{\varphi}^{(\nu)}$. Set

$$
v(\epsilon):=G_{0, \nu}(\varphi, \varphi, E+i \epsilon), \quad w(\epsilon):=G_{0, \nu}(\psi, \psi, E+i \epsilon),
$$

and note that

$$
G_{\lambda, \nu}(\varphi, \varphi, E+i \epsilon)=\frac{v(\epsilon)}{1-\lambda^{2} v(\epsilon) w(\epsilon)} .
$$

By (2.9), $E \in \mathcal{S}_{\varphi}\left(H_{\lambda, \nu}\right)$ for some $\lambda \neq 0$ and $\lim _{\epsilon \backslash 0} \operatorname{Im}_{\lambda, \nu}(\varphi, \varphi, E+i \epsilon)=\infty$ is equivalent to

$$
\lim _{\epsilon \searrow 0} \operatorname{Im} \frac{1}{\frac{1}{v(\epsilon)}-\lambda^{2} w(\epsilon)}=\infty
$$

Since for any $z \in \mathbb{C}$,

$$
\left|\operatorname{Im} \frac{1}{z}\right|=\left|\frac{-\operatorname{Im} z}{|z|^{2}}\right| \leq \frac{1}{|\operatorname{Im} z|}
$$

we observe that $\left|\operatorname{Im} z^{-1}\right| \rightarrow \infty$ implies $\operatorname{Im} z \rightarrow 0$ and hence also $\operatorname{Re} z \rightarrow 0$. This leads to

$$
\frac{1}{v(\epsilon)}-\lambda^{2} w(\epsilon)=: h(\epsilon) \rightarrow 0
$$

as $\epsilon \rightarrow 0$.

Suppose that $\sup _{n}\left|v\left(\epsilon_{n}\right)\right|<\infty$ along some sequence $\epsilon_{n} \downarrow 0$. Since

$$
\frac{v(\epsilon)}{w(\epsilon)}=\frac{\lambda^{2} v(\epsilon)^{2}}{1-v(\epsilon) h(\epsilon)}
$$

we have (recall (2.8))

$$
\infty=\lim _{n \rightarrow \infty}\left|\operatorname{Re}\left(\sqrt{\frac{v\left(\epsilon_{n}\right)}{w\left(\epsilon_{n}\right)}}\right)\right| \leq \limsup _{n \rightarrow \infty} \sqrt{\left|\frac{v\left(\epsilon_{n}\right)}{w\left(\epsilon_{n}\right)}\right|}=|\lambda| \limsup _{n \rightarrow \infty}\left|v\left(\epsilon_{n}\right)\right|<\infty,
$$

which is a contradiction. Hence $\lim _{\epsilon \backslash 0}|v(\epsilon)|=\infty$. This fact and (2.11) yield that

$$
\lim _{\epsilon \searrow 0} w(\epsilon)=0 .
$$


For $E \in \mathbb{R} \backslash \sigma\left(H_{S}\right)$ we set

$$
d(E)=G_{0}\left(\delta_{\ell}, \delta_{\ell}, E\right) G_{0}\left(\delta_{r}, \delta_{r}, E\right)-G_{0}\left(\delta_{\ell}, \delta_{r}, E\right) G_{0}\left(\delta_{r}, \delta_{\ell}, E\right) .
$$

Let

$$
\mathcal{N}:=\left\{E \in \mathbb{R} \backslash \sigma\left(H_{S}\right) \mid G_{0}\left(\delta_{\ell}, \delta_{\ell}, E\right) G_{0}\left(\delta_{r}, \delta_{r}, E\right) G_{0}\left(\delta_{\ell}, \delta_{r}, E\right) d(E)=0\right\} \cup \sigma\left(H_{S}\right)
$$

The set $\mathcal{N}$ is finite.

Lemma 2.3 (1) $C_{\varphi, \psi}^{(0)} \subset \mathcal{N}$.

(2) For $\nu \neq 0$,

$$
C_{\chi_{\ell}, \delta_{\ell}}^{(\nu)} \backslash \mathcal{N}=\left\{E \in \mathbb{R}\left|\lim _{\epsilon \searrow 0}\right| G_{0}\left(\chi_{\ell}, \chi_{\ell}, E+i \epsilon\right) \mid=\infty, \lim _{\epsilon \searrow 0} G_{0}\left(\chi_{r}, \chi_{r}, E+i \epsilon\right)=\frac{G_{0}\left(\delta_{\ell}, \delta_{\ell}, E\right)}{\nu^{2} d(E)}\right\} .
$$

(3) For $\nu \neq 0$,

$$
C_{\delta_{\ell}, \chi_{\ell}}^{(\nu)} \backslash \mathcal{N}=\left\{E \in \mathbb{R}\left|\lim _{\epsilon \searrow 0}\right| G_{0}\left(\chi_{\ell}, \chi_{\ell}, E+i \epsilon\right) \mid=0, \lim _{\epsilon \backslash 0} G_{0}\left(\chi_{r}, \chi_{r}, E+i \epsilon\right)=\frac{1}{\nu^{2} G_{0}\left(\delta_{r}, \delta_{r}, E\right)}\right\} .
$$

Proof. We will deal with the case $\varphi=\chi_{\ell}, \psi=\delta_{\ell}$, the other case is similar. Note that

$$
G_{0, \nu}\left(\chi_{\ell}, \chi_{\ell}, E+i \epsilon\right)=G_{0}\left(\chi_{\ell}, \chi_{\ell}, E+i \epsilon\right),
$$

and that by Lemma 2.1,

$G_{0, \nu}\left(\delta_{\ell}, \delta_{\ell}, E+i \epsilon\right)=G_{0}\left(\delta_{\ell}, \delta_{\ell}, E+i \epsilon\right)+\nu^{2} \frac{G_{0}\left(\chi_{r}, \chi_{r}, E+i \epsilon\right) G_{0}\left(\delta_{\ell}, \delta_{r}, E+i \epsilon\right) G_{0}\left(\delta_{r}, \delta_{\ell}, E+i \epsilon\right)}{1-\nu^{2} G_{0}\left(\chi_{r}, \chi_{r}, E+i \epsilon\right) G_{0}\left(\delta_{r}, \delta_{r}, E+i \epsilon\right)}$.

Part (1) is now obvious and simple algebra yields Part (2).

We are now ready to complete the proof of Theorem 1.4. We shall deal with the case $\varphi=\chi_{\ell}$. The argument is identical in the case $\varphi=\delta_{\ell}$.

Let

$$
\Omega_{\nu}:=C_{\chi_{\ell}, \delta_{\ell}}^{(\nu)} \backslash \mathcal{N}
$$

By Lemma 2.2, the singular part of $\bar{\mu}_{\chi \ell}^{(\nu)} \uparrow_{\mathbb{R} \backslash \mathcal{N}}$, is concentrated on $\Omega_{\nu}$. Our strategy is to show that

$$
\mathbf{1}_{\Omega_{\nu}}\left[H_{\lambda, \nu}\right]=0
$$

for a.e. $\lambda, \nu$. This implies the statement since

$$
\bar{\mu}_{\chi \ell}^{(\nu)}\left(\Omega_{\nu}\right)=\int_{\mathbb{R}}\left\|\mathbf{1}_{\Omega_{\nu}}\left[H_{\lambda, \nu}\right] \chi_{\ell}\right\|^{2} d \lambda .
$$


Let

$\mathcal{A}:=\left\{E \in \mathbb{R}\left|\lim _{\epsilon \backslash 0}\right| G_{0}\left(\chi_{\ell}, \chi_{\ell}, E+i \epsilon\right) \mid=\infty, \lim _{\epsilon \backslash 0} G_{0}\left(\chi_{r}, \chi_{r}, E+i \epsilon\right)\right.$ exists and is finite and non-zero $\}$ $\mathcal{L}(\mathcal{A})=0$ and by Lemma $2.3, \Omega_{\nu} \subset \mathcal{A}$ for all $\nu$. We claim that for all $\lambda$,

$$
\begin{aligned}
& \bar{\mu}_{\chi_{r}}^{(\lambda)}(\mathcal{A})=\int_{\mathbb{R}} \mu_{\chi_{r}}^{(\lambda, \nu)}(\mathcal{A}) d \nu=0, \\
& \bar{\mu}_{\delta_{r}}^{(\lambda)}(\mathcal{A})=\int_{\mathbb{R}} \mu_{\delta_{r}}^{(\lambda, \nu)}(\mathcal{A}) d \nu=0 .
\end{aligned}
$$

To establish these relations, define $C_{\varphi, \psi}^{(\lambda)}$ by (2.10) with $\lambda$ replacing $\nu$ and $\varphi, \psi \in\left\{\chi_{r}, \delta_{r}\right\}, \varphi \neq \psi$. By Lemma 2.2 and the remark after it, the singular parts of the measures $\bar{\mu}_{\chi_{r}}^{(\lambda)}, \bar{\mu}_{\delta_{r}}^{(\lambda)}$ are concentrated on the set

$$
\mathcal{A}^{\prime}=\left\{E \in \mathbb{R}\left|\lim _{\epsilon \searrow 0}\right| G_{0}\left(\chi_{r}, \chi_{r}, E+i \epsilon\right) \mid=\infty\right\} \cup\left\{E \in \mathbb{R} \mid \lim _{\epsilon \searrow 0} G_{0}\left(\chi_{r}, \chi_{r}, E+i \epsilon\right)=0\right\} .
$$

Since $\mathcal{A} \cap \mathcal{A}^{\prime}=\emptyset$, the relations (2.13) follow. As a consequence of (2.13), for a given $\lambda$ we have

$$
\mathbf{1}_{\Omega_{\nu}}\left[H_{\lambda, \nu}\right] \delta_{r}=\mathbf{1}_{\Omega_{\nu}}\left[H_{\lambda, \nu}\right] \chi_{r}=0 \quad \text { for a.e. } \nu .
$$

It follows that for a.e. $\nu$

$$
H_{\lambda, \nu} \psi=H_{\lambda, 0} \psi
$$

where $\psi \in \operatorname{Ran} \mathbf{1}_{\Omega_{\nu} \cap[-M, M]}\left[H_{\lambda, \nu}\right]$ and $M>0$. An application of the functional calculus gives that for a.e. $\nu$ and for any bounded Borel function $f$,

$$
f\left(H_{\lambda, \nu}\right) \uparrow \operatorname{Ran} \mathbf{1}_{\Omega_{\nu}}\left[H_{\lambda, \nu}\right]=f\left(H_{\lambda, 0}\right) \uparrow \operatorname{Ran} \mathbf{1}_{\Omega_{\nu}}\left[H_{\lambda, \nu}\right] .
$$

In particular, for a.e. $\nu$ and all $\psi \in \operatorname{Ran} \mathbf{1}_{\Omega_{\nu}}\left[H_{\lambda, \nu}\right]$,

$$
\psi=\mathbf{1}_{\Omega_{\nu}}\left(H_{\lambda, 0}\right) \psi
$$

Note that

$$
H_{\lambda, 0}=h_{\lambda} \oplus H_{r}
$$

where

$$
h_{\lambda}:=H_{\ell}+H_{S}+\lambda\left[\left(\chi_{\ell}, \cdot\right) \delta_{\ell}+\left(\delta_{\ell}, \cdot\right) \chi_{\ell}\right]
$$

acts on $\mathcal{H}_{\ell} \oplus \mathcal{H}_{S}$. Lemma 2.3 (1) implies that for all $\nu$

$$
\int_{\mathbb{R}} \mu_{\chi \ell}^{(\lambda, 0)}\left(\Omega_{\nu}\right) d \lambda=\int_{\mathbb{R}} \mu_{\delta_{\ell}}^{(\lambda, 0)}\left(\Omega_{\nu}\right) d \lambda=0
$$

Since $h_{\lambda}=H_{S}$ on the orthogonal complement (in $\mathcal{H}_{\ell} \oplus \mathcal{H}_{S}$ ) of the cyclic space spanned by $h_{\lambda}$ and $\left(\chi_{\ell}, \delta_{\ell}\right)$, we derive that

$$
\mathbf{1}_{\Omega_{\nu}}\left(h_{\lambda}\right)=0 \quad \text { for a.e. } \lambda .
$$


The singular part of the spectral measure of $H_{r}$ and $\chi_{r}$ is concentrated on the set

$$
\left\{E \in \mathbb{R} \mid \lim _{\epsilon \backslash 0} \operatorname{Im} G_{0}\left(\chi_{r}, \chi_{r}, E+i \epsilon\right)=\infty\right\}
$$

and so $\mathbf{1}_{\Omega_{\nu}}\left(H_{r}\right)=0$. Hence, for a.e $\lambda, \nu$ and any $\psi \in \operatorname{Ran} \mathbf{1}_{\Omega_{\nu}}\left[H_{\lambda, \nu}\right]$,

$$
\psi=\mathbf{1}_{\Omega_{\nu}}\left(H_{\lambda, 0}\right) \psi=0,
$$

and (2.12) follows.

\section{References}

[AJJP1] Aschbacher, W., Jakšić, V., Pautrat, Y., Pillet, C.-A.: Topics in non-equilibrium quantum statistical mechanics. In Open Quantum System III. Recent Developments. S. Attal, A. Joye and C.-A. Pillet editors. Lecture Notes in Mathematics 1882, 1, Springer, New York, 2006.

[AJPP2] Aschbacher, W., Jakšić, V., Pautrat, Y., Pillet, C.-A.: Transport properties of quasi-free fermions. J. Math. Phys. 48, 032101 (2007).

[BFS] Bach, V., Fröhlich, J., Sigal, I.: Quantum electrodynamics of confined non-relativistic particles. Adv. Math. 137, 299 (1988).

[DJ] Dereziński J., Jakšić, V.: Spectral theory of Pauli-Fierz operators. J. Func. Anal. 180, 243 (2001).

[J] Jakšić, V.: Topics in spectral theory. In Open Quantum Systems I. The Hamiltonian Approach. S. Attal, A. Joye and C.-A. Pillet editors. Lecture Notes in Mathematics 1880, 235, Springer, New York, 2006.

[JKP] Jakšić, V., Kritchevski, E., Pillet, C.-A.: Mathematical theory of the Wigner-Weisskopf atom. In Large Coulomb systems. J. Dereziński and H. Siedentop editors. Lecture Notes in Physics 695, 145, Springer, New York, 2006.

[N] Nenciu, G.: Independent electrons model for open quantum systems: Landauer-Büttiker formula and strict positivity of the entropy production. J. Math. Phys. 48, 033302 (2007).

[S] Simon, B.: Spectral analysis and rank one perturbations and applications. In CRM Lecture Notes, 8, (J. Feldman, R. Froese, L. Rosen eds), 109, Amer. Math. Soc., Providence, RO, 1995.

[SW] Simon, B., Wolff, T.: Singular continuous spectrum under rank one perturbations and localization for random Hamiltonians. Commun. Pure App. Mathem. 49 (1986), 75. 